\title{
Paternidade contemporânea: levantamento da produção acadêmica no período de 2000 a 2007
}

\author{
Carmen Lúcia Carvalho de Souza ${ }^{1}$ \\ Universidade Comunitária Regional de Chapecó, Chapecó-SC, Brasil \\ Silvia Pereira da Cruz Benetti \\ Universidade do Vale do Rio dos Sinos, São Leopoldo-RS, Brasil
}

\begin{abstract}
Resumo: Este estudo teve como objetivo verificar a incidência de artigos internacionais e nacionais sobre o tema paternidade no período de 2000 a 2007 indexados nas bases de dados: LILACS, SciELO, Web of Science, MEDLINE, Redalyc. Um total de 2.205 trabalhos foi identificado, sendo analisados 353 artigos em relação ao ano de publicação, país, método e temática. Os resultados apontam para significativa produção internacional e nacional, destacando-se EUA, Inglaterra e Brasil. Verificou-se que o tema paternidade é foco importante para a compreensão das relações familiares, questão fundamental para a implantação de políticas públicas de apoio às famílias em diferentes contextos.
\end{abstract}

Palavras-chave: paternidade, pais, família, homens.

\section{Contemporary fatherhood: review of academic production between 2000 and 2007}

\begin{abstract}
This study aimed to verify the incidence of national and international studies on fatherhood between 2000 and 2007 indexed in the following databases: LILACS, SciELO, Web of Science, MEDLINE, Redalyc. A total of 2205 articles were identified and 353 were analyzed regarding year of publication, country, method and thematic. Results reveal significant national and international production with the USA, England and Brazil presenting outstanding positions. It was verified that fatherhood is an important topic to understand family relations and essential issue in the implementation of public policies to support families in different contexts.
\end{abstract}

Keywords: fatherhood, parents, family, men.

\section{Paternidad contemporánea: levantamiento de la producción académica en el período de 2000 a 2007}

\begin{abstract}
Resumen: Este estudio tuvo como objetivo comprobar la ocurrencia de artículos internacionales y nacionales sobre el tema paternidad en el período de 2000 a 2007, indexados en las bases de datos: LILACS, SciELO, Web of Science, MEDLINE, Redalyc. Un total de 2205 trabajos fue identificado, siendo analizados 353 artículos en relación a lo año de publicación, país, método y temática. Los resultados apuntan para significativa producción internacional y nacional, destacándose EUA, Inglaterra y Brasil. Se ha comprobado que el tema de la paternidad es foco importante para comprensión de las relaciones familiares, cuestión fundamental para implantación de políticas públicas de apoyo a las familias en distintos contextos.
\end{abstract}

Palabras clave: paternidad, padres, familia, hombres.

O conceito de paternidade tem se modificado ao longo das épocas, refletindo as alterações no contexto socioeconômico e cultural das sociedades. A observação do exercício da paternidade, sob o enfoque histórico, demonstra que as características dos papéis e interações familiares sofreram transformações na sociedade ocidental, desde o modelo patriarcal, entendido como um sistema de organização familiar centrada na figura masculina (Narvaz \& Koller, 2006), até a multifacetada sociedade pós-moderna quando surgem novos formatos de família (Lamb, 1997). A paternidade deixou de incluir somente o papel limitado à figura de provedor para também abarcar atitudes de maior envolvimento e contato afetivo com os filhos (Balancho, 2004; Brito, 2005), sendo estas mudanças associadas a um novo conjunto de expectativas, crenças e atitudes de cada gênero no contexto familiar (Brasileiro, Jablonski, \& Féres-Carneiro, 2002). Estas transformações também se refletiram no interesse das pesquisas em identificar

\footnotetext{
1 Endereço para correspondência:

Profa. Ms. Carmen Lúcia Carvalho de Souza. Av. Senador Attílio Fontana, 591-E, Caixa Postal 1141. CEP 89809-000. Chapecó-SC, Brasil. E-mail.
} carmen_1cs@hotmail.com e compreender o impacto destas mudanças nas relações familiares, especificamente no próprio pai (Lamb, 1997).

Avaliando-se a produção acadêmica da década de 1970, percebe-se que as pesquisas sobre a paternidade ainda eram dominadas pelos estudos sobre mulheres no contexto familiar. Somente nos anos 1980 as temáticas relacionadas à construção social da masculinidade e sua influência no papel paterno surgiram de forma mais consistente, indicando uma participação mais efetiva da figura paterna no cotidiano familiar (Hennigen \& Guareschi, 2002). O novo pai passou a ser representado na literatura, televisão, filmes e revistas como mais envolvido emocionalmente, mais participativo e comprometido com suas crianças e tão capaz quanto as mães na educação dos filhos (Wall \& Arnold, 2007). Assim, identificou-se o desejo masculino de experiências mais afetivas, impulsionando-o ao maior envolvimento com seus filhos (Bustamante, 2005; Gomes \& Resende, 2004). Contudo, mesmo que a caracterização do novo pai incluísse papéis mais participativos em relação ao envolvimento masculino no cuidado dos filhos, também subsistiam no imaginário social marcas da estrutura tradicional do pai provedor. 
Neste sentido, a nova consciência dos pais com relação à necessidade de maior envolvimento com os filhos e menor investimento na carreira profissional encontrava muitos entraves na prática cotidiana masculina, revelando que a transformação dos valores não segue o ritmo das mudanças sociais. Na construção de um novo modelo de paternidade, os homens ainda se mostram menos frequentemente envolvidos com seus filhos menores do que as mães (Tudge e cols., 2000), sendo que as mudanças permanecem associadas às influências mais específicas de determinantes pessoais e contextuais. Como observam os pesquisadores Silva e Piccinini (2004), esta nova concepção sobre a paternidade está mais presente no discurso dos estudiosos e da sociedade em geral do que inserida como prática permanente dos pais.

Entretanto, é inegável que as transformações do papel masculino na família e o maior envolvimento masculino no cuidado e responsabilidade para com os filhos foram uma das grandes mudanças ocorridas nas sociedades ocidentais no século XX. Neste sentido, Cabrera e cols. (2000), destacaram que o ingresso da mulher na força de trabalho, o aumento de famílias monoparentais e o crescente envolvimento paterno nas famílias tradicionais foram aspectos determinantes nas transformações das concepções de família, parentalidade e das relações familiares em geral. Para estes autores, compreender estas transformações para integrá-las às teorias, às práticas metodológicas e às políticas públicas de apoio às famílias é uma ação fundamental de trabalho e pesquisa. Desse modo, questionam quais aspectos seriam destacados na paternidade a partir do século XXI.

Diante de tais considerações, este artigo teve como objetivo verificar a produção de artigos acadêmicos nacionais e internacionais abordando o tópico paternidade, a partir do ano 2000. Estes artigos foram analisados em relação ao período, metodologia e temática abordada, visando identificar as tendências e particularidades dos tópicos investigados sobre a paternidade.

\section{Método}

Num primeiro momento, foi identificada a incidência dos estudos científicos sobre o tema paternidade no período de 2000 a 2007, por meio de levantamento dos artigos internacionais e nacionais indexados nas bases de dados LILACS (Literatura Latino-Americana e do Caribe de Informação em Ciências da Saúde) e SciELO (Scientific Electronic Library Online). Além destas, foram consultadas também as bases Web of Science, MEDLINE (Medical Literature Analysis and Retrieval System Online), Redalyc (Red de Revistas Científicas de América Latina, el Caribe, Espanã y Portugal). Neste levantamento utilizaram-se os descritores fatherhood, father, fathers e paternidade. Inicialmente foram encontradas 2.205 referências. Com a exclusão de teses, capítulos de livros, livros, guias, comentários, resenhas, informativos governamentais, artigos duplicados e artigos que se afastavam da temática paternidade, reduziu-se a análise para 353 resumos de artigos.
Estes trabalhos foram classificados segundo: (a) Número de artigos publicados por ano, nacionais e internacionais; (b) País da revista; (c) Metodologia: estudos quantitativos, qualitativos e teóricos; (d) Temática do trabalho. Neste estudo foram considerados artigos teóricos as revisões de literatura, meta-análise e revisões sistemáticas; artigos quantitativos, trabalhos baseados em hipóteses claramente indicadas e variáveis com definição operacional; artigos qualitativos, dados descritivos obtidos mediante contato direto e interativo do pesquisador com a situação objeto de estudo (Neves, 1996). Para a análise da temática dos trabalhos foram utilizadas as categorias de Pleck (1997), para quem os estudos sobre a paternidade abordam:

(1) Características da paternidade: temas sobre a descrição, experiências e características do envolvimento paterno, ciclo vital (adolescência, adulto jovem, meia-idade);

(2) Determinantes da paternidade: estudos sobre fatores associados ao envolvimento masculino: (a) Características das crianças e variáveis sociodemográficas do pai; (b) Motivação: temas concernentes à influência da história de vida, personalidade, características, crenças dos pais; (c) Habilidades e confiança: temas relacionados à competência no cuidado da criança, (d) Suporte social: relacionamento conjugal e ciclo de vida familiar (divórcio, famílias reconstituídas, adoção); (e) Fatores institucionais, históricos, políticos e culturais: temas enfocando programas institucionais e aspectos contextuais da vivência em sociedade, influências e implicações;

(3) Consequências da paternidade: (a) Consequências para o pai: temas vinculados aos efeitos da paternidade para o próprio pai; (b) Consequências para o filho: influência no desenvolvimento infantil. Assim, estabeleceu-se um processo de análise e classificação sistemática, incluindo cada artigo em uma categoria e subcategoria. Apesar da diversidade do foco de alguns artigos, optou-se pela classificação na qual prevalecesse o tema central ou dominante.

\section{Resultados}

No período de 2000 a 2007 foram encontrados 263 artigos internacionais e 90 artigos nacionais que abordavam o tópico da paternidade. As publicações internacionais englobaram 136 artigos qualitativos, 85 teóricos e 42 quantitativos. A maior produção teórica internacional foi em 2003, com 23 artigos, o método qualitativo apresentou mais alta incidência em 2004, com 24 artigos. As publicações nacionais totalizaram 50 artigos qualitativos, 31 artigos teóricos e 9 artigos quantitativos. No ano de 2002 e 2006 houve a maior incidência de artigos teóricos (8) e de artigos qualitativos (14). Portanto, houve um menor número de trabalhos quantitativos, indicando que as pesquisas privilegiaram o conhecimento processual de experiências sobre a paternidade. A Tabela 1 descreve as publicações selecionadas de acordo com ano de publicação, nacionalidade e método. 
Tabela 1

Publicações nacionais e internacionais no período de 20002007 segundo ano de publicação, nacionalidade e delineamento metodológico

\begin{tabular}{|c|c|c|c|c|c|c|c|}
\hline \multirow[b]{2}{*}{ Ano } & \multicolumn{2}{|c|}{ Teórico } & \multicolumn{2}{|c|}{ Qualitativo } & \multicolumn{2}{|c|}{ Quantitativo } & \\
\hline & 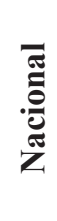 & 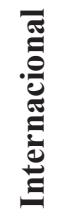 & 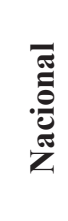 & 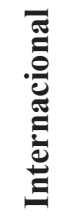 & 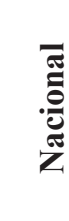 & 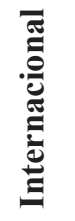 & Total \\
\hline 2000 & 2 & 13 & 5 & 7 & 0 & 4 & 21 \\
\hline 2001 & 4 & 2 & 1 & 10 & 1 & 6 & 34 \\
\hline 2002 & 8 & 0 & 7 & 8 & 1 & 6 & 30 \\
\hline 2003 & 5 & 23 & 6 & 11 & 0 & 3 & 48 \\
\hline 2004 & 2 & 9 & 9 & 24 & 0 & 9 & 53 \\
\hline 2005 & 2 & 12 & 4 & 18 & 1 & 6 & 43 \\
\hline 2006 & 8 & 14 & 14 & 37 & 4 & 6 & 83 \\
\hline 2007 & 0 & 12 & 4 & 21 & 2 & 2 & 41 \\
\hline Total & 31 & 85 & 50 & 136 & 9 & 42 & 353 \\
\hline
\end{tabular}

Em relação aos países, apenas EUA, Inglaterra e Brasil tiveram publicações em todos os anos do período avaliado. Os EUA com 157 artigos e a Inglaterra com 50 estudos destacam-se na produção internacional. Ainda assim, o Brasil produziu 90 publicações durante o período, indicando significativa contribuição ao tema. De fato, em 2006 a produção nacional de 26 artigos foi similar à verificada nos Estados Unidos, que foi de 30 artigos naquele ano.

\section{Análise por Categoria}

$\mathrm{Na}$ análise temática classificaram-se os artigos quanto às características $(n=104,30 \%)$, aos determinantes $(n=164$, $46 \%)$ e às consequências da paternidade $(n=85,24 \%)$. Em relação ao total de artigos internacionais, o maior número de pesquisas incluiu temáticas sobre a paternidade adulta $(n=45$, $17 \%$ ), ao buscar compreender o "novo pai" contemporâneo e temáticas políticas e sociais $(n=61,24 \%)$ referentes a ele. O Brasil manteve uma produção significativa, concentrando a maior parte dos estudos sobre a paternidade na adolescência $(n=18,20 \%)$ e nas temáticas políticas e sociais $(n=21$, $16 \%)$.

\section{Características da paternidade}

Nesta categoria foram incluídos os temas referentes à descrição, às experiências e características do envolvimento paterno, à compreensão da paternidade, ao ciclo vital (adolescência, adulto jovem, meia-idade). Do total de 104 artigos analisados nesta categoria, 45 tratavam sobre a paternidade em relação à adolescência e 59 em relação à idade adulta. Deste total, 32 trabalhos eram de produção nacional. As subcategorias são descritas a seguir:

(1) Paternidade adulta: nesta subcategoria constatase o grande interesse internacional na paternidade adulta
( $n=45,17 \%)$, focalizando análises da paternidade em relação a questões macropolíticas, históricas e sociais, abordando a experiência de transição e identificando as mudanças ocorridas com relação ao envolvimento paterno (Peterson \& Steinmetz, 2000; Seward, 2003) até aspectos da paternidade em grupos vulneráveis, tais como, usuários de drogas (Arenas \& Greif, 2000). Os cientistas sociais buscaram uma compreensão dinâmica do histórico da paternidade (Lamb, 2000) e da atual preocupação dos homens em serem "bons pais" (Perala-Littunen, 2007), discutindo a disponibilidade dos pais (Gray, 2006), além da responsabilidade paterna (Marsiglio, Hutchinson, \& Cohan, 2000). Destacam-se análises sobre contradições nas transformações da paternidade que não se traduzem em um maior envolvimento no cuidado com os filhos (McVeigh, Baafi, \& Williamson, 2002). As publicações nacionais apresentaram produção importante nesta área $(n=14,16 \%)$, incluindo trabalhos sobre a caracterização da paternidade quanto aos aspectos político-sociais, questões de gênero e abordagens psicanalíticas, destacando-se trabalhos sobre o envolvimento paterno durante a gestação (Tudge, Piccinini, Silva, Gonçalves, \& Lopes, 2004). Outras temáticas foram questões políticas, de cidadania/sociais e de gênero que implicam a deserção paterna (Thurler, 2004), o significado da paternidade na adoção (Andrade, Costa, \& Rossetti-Ferreira, 2006), a avaliação da paternidade na década passada e o surgimento de um pai mais participativo no lar (Dermott, 2003).

(2) Paternidade adolescente: A paternidade na adolescência recebeu menor destaque pela comunidade científica internacional $(n=27,10 \%)$ em relação à nacional $(n=18$, $20 \%$ ). Em geral, os resumos mencionavam dificuldades socioeconômicas relacionadas à paternidade e delinquência (Wei, Loeber, \& Stouthamer-Loeber, 2002), sob o enfoque das questões políticas/sociais, avaliando as necessidades dos jovens ao se tornarem pais (Bishop, Gay, Ledesma, \& Garofalo, 2005). Já nas pesquisas brasileiras, houve número maior de publicações, investigando o significado da paternidade para os jovens pais e como as relações de gênero contribuem para tornar os adolescentes mais vulneráveis à gravidez (Almeida \& Hardy, 2007). Os temas abordados retrataram a paternidade no adolescente brasileiro de baixa renda como um fator de consolidação da imagem masculina e responsabilidade adulta (Cabral, 2003). O fenômeno da paternidade surge como elemento motivador para a união conjugal juvenil ativando no adolescente as funções de prover e cuidar da criança (Dias \& Aquino, 2006).

Em suma, a incidência de temas relacionados às características da paternidade na produção internacional e nacional confirmou o interesse dos pesquisadores em aprofundar o conhecimento sobre a questão. Percebe-se no Brasil um maior número de publicações sobre a paternidade adolescente, considerando os fatores de risco e proteção. Nas pesquisas brasileiras de 2002, por exemplo, mais de 30\% dos trabalhos abordaram a paternidade na adolescência e o significado atribuído ao papel de pai (Trindade \& Menandro, 2002), refletindo sobre a gravidez enquanto um problema 
social (Heilborn e cols., 2002). No âmbito internacional a maior parte dos artigos discute a paternidade adulta e o novo modelo de pai mais envolvido na relação com seus filhos, embora este ainda não se apresente como um padrão geral, permanecendo referências ao modelo patriarcal nas relações de gênero (Gaertner, Spinrad, Eisenberg, \& Greving, 2007).

\section{Determinantes do envolvimento}

Nesta categoria 170 artigos foram analisados e divididos em subcategorias de acordo com as temáticas.

(1) Características da criança e variáveis sociodemográficas do pai: nesta subcategoria foram analisados 32 artigos, sendo a maioria internacionais. Principalmente a partir de 2001, o foco dos artigos voltou-se para compreensão da paternidade frente às dificuldades financeiras encontradas em famílias de baixa renda (Aronson, Whitehead, \& Baber, 2003), as condições de vida que contribuem para a depressão em pais com salário baixos (Anderson, Kohler, \& Letiecq, 2005). Outros artigos enfocaram o desemprego que leva à permanência do homem em casa e à perda do papel de provedor (Thomas \& Bailey, 2006), os pais sem-teto e sua vivência junto aos filhos em abrigos (Schindler \& Coley, 2007) e o funcionamento das famílias em que os pais estão presos (Hairston, 2002). As mudanças socioculturais se refletiram nas pesquisas que examinam as implicações da paternidade e práticas paternas do pai empregado que trabalha no lar (Halford, 2006), o uso da licença parental (Bygren \& Duvander, 2006) e o cuidado direto dos pais com filhos com problemas de saúde (Davies e cols., 2004). No Brasil, as questões específicas da saúde do homem relacionadas ao trabalho e à paternidade (Rocha \& Debert-Ribeiro, 2001), e à transmissão geracional das dificuldades afetivas decorrentes da pobreza, foram analisadas considerando as características pessoais dos membros das famílias (Bigras \& Paquette, 2000), apenas um artigo tratou da experiência de pais com filhos prematuros (Tronchin \& Tsunechiro, 2006). A menor produção nacional em relação à internacional indica a necessidade do incremento da atenção dos pesquisadores para questões específicas associadas à paternidade em situação de risco, como filhos doentes, questões sociais, dificuldades econômicas, condições de trabalho ou desemprego e sua repercussão impeditiva no exercício da paternidade.

(2) Motivação: somente 14 artigos abordaram histórias de vida, personalidade, características, crenças dos pais associadas à motivação e ao interesse no cuidado dos filhos. No Brasil esta é a categoria com o mais baixo índice de artigos indexados $(n=3,3 \%)$. Os resumos identificados discutiram a construção de gênero e da paternidade transmitida nos livros infantis (Anderson \& Hamilton, 2005) e a influência da experiência paterna com seus próprios pais no envolvimento com seus filhos (Masciadrelli, Pleck, \& Stueve, 2006). Os artigos nacionais são limitados nesta categoria e abordam o interesse na paternidade e no aleitamento materno (Serafim \& Lindsey, 2002), bem como questões de motivação e infertilidade (Farinati, Rigoni, \& Müller, 2006).
(3) Habilidades e confiança: ao todo nove trabalhos foram classificados nesta categoria. Os artigos internacionais $(n=5,2 \%)$ e nacionais $(n=6,7 \%)$ complementam as discussões da categoria características da paternidade, refletindo sobre a participação paterna no cuidado direto com os filhos, demonstrando que no trabalho fora do lar os homens são mais "experts" do que nas atividades de paternagem (Pohlman, 2005). Sugere-se que os homens desenvolvam esta capacidade através do relacionamento com suas esposas e contato com suas crianças (Madsen, Lind, \& Munck, 2007). No Brasil, observa-se que os resumos versam sobre a relevância da presença masculina no desenvolvimento dos filhos, avaliando os fatores determinantes nesta função e na escolha de estratégias educativas (Bem \& Wagner, 2006).

(4) Suporte social: nesta categoria identificaram-se 31 artigos internacionais e nacionais (ambos com 8\%) voltados para questões envolvendo pais não-residentes e o relacionamento com seus filhos. Os estudos que tratam dos pais divorciados acompanham as mudanças culturais das últimas três décadas, caracterizadas pelo aumento crescente no número de divórcios (Gupta, Smock, \& Manning, 2004). Os aspectos do divórcio e o apoio para a criança (Manning \& Smock, 2000) foram analisados nas questões de custódia, visitas e decisões de pensões alimentícias (Skevik, 2006). Também foi discutido o nível de envolvimento dos pais que habitam e dos que não co-habitam com suas crianças (Nelson, 2004) e daqueles que optam não viver com seus filhos (Kiernan, 2006), além da análise sobre a inevitável limitação da paternidade na não-custódia (Baum, 2004). Outro ponto envolve os pais não-biológicos e sua proximidade com crianças adotivas (Eggebeen \& Knoester, 2001), assim como a relação dos padrastos com seus enteados (Marsiglio \& Hinojosa, 2007). Alguns artigos analisaram as famílias homossexuais, concluindo que o gênero é menos importante para o relacionamento e cuidado da criança (Lubbe, 2007). A publicação brasileira focalizou a construção e manutenção do vínculo afetivo entre pais e filhos após a separação, estudando o exercício da paternidade à distância e sua implicação para os filhos (Dantas, Jablonski, \& Féres-Carneiro, 2004). Os artigos também revisaram temas ligados ao apoio emocional dos pais às suas esposas durante a gestação e trabalho de parto (Motta \& Crepaldi, 2005) e a homoparentalidade como uma entre tantas estruturas familiares (Passos, 2005).

(5) Fatores institucionais, históricos, políticos e sociais: esta categoria apresenta o maior índice de publicações, 81 artigos, sendo $23 \%(n=61)$ de trabalhos internacionais e $16 \%(n=14)$ de estudos nacionais, relacionados aos processos de mudanças sociais que alteraram o contexto cultural e social em que as crianças estão se desenvolvendo (Marsiglio, Day, \& Lamb, 2000; Walker \& McGraw, 2000), com modificações significativas em relação à prática da paternidade dos anos 1950 (LaRossa, 2004). As publicações destacaram a distribuição de renda e as oportunidades de emprego considerando seus efeitos sobre a paternidade (Whitehouse, 2002), as políticas de incentivo à participação dos pais no cuidado 
das crianças (Cabrera \& Peters, 2000; Joseph, 2006), além de investigações acerca da imigração e seus efeitos sobre a paternidade enquanto esforço de adaptação à nova cultura (Gonzalez-Lopez, 2004). Os resumos referem estudos sobre a efetividade dos programas durante a gravidez das esposas (Kao \& Long, 2004), famílias de baixa renda (Anderson, Kohler, \& Letiecq, 2002), pais separados (Bloomer \& Sipe, 2003) e pais presos (MacMillan, 2005). A análise de edições de periódicos dedicados à família mostra como a cultura contemporânea mantém o papel de envolvimento do "novo pai", ainda que na prática a mãe continue sendo a principal cuidadora (Wall \& Arnold, 2007). A produção brasileira abordou a paternidade no período de 1940-90 (Santos, Caldana, \& Biasoli-Alves, 2001) até os novos caminhos para a paternidade brasileira no século XXI (Thurler, 2006). As pesquisas também buscaram conhecer as contradições presentes no discurso social acerca de homens e mulheres na sociedade atual que repercutem nas expectativas da atividade parental e apontam a importância da mídia na construção da identidade paterna (Hennigen \& Guareschi, 2002). Apontouse que a paternidade é construída pela qualidade da relação com a parceira e a experiência como filho, e não a partir do laço biológico com a criança, além de estar baseada na noção de provedor como condição necessária para uma relação afetiva com os filhos (Bustamante, 2005). Também se observaram artigos sobre a adoção por parte de homossexuais (Futino \& Martins, 2006) e a vontade de ter filhos em homens portadores de HIV (Paiva, Lima, Santos, Ventura-Filipe, \& Segurado, 2002). Alguns resumos apontaram a exclusão do pai na participação do pré-natal em programas de saúde e no parto (Carvalho, 2003).

Destaca-se a preocupação dos trabalhos em abordar as questões político-sociais em diversos grupos sociais, dando ênfase à necessidade de apoio e suporte tanto ao homem como às famílias no tocante ao incentivo do envolvimento paterno. Por exemplo, nos artigos internacionais do ano 2000, mais de $45 \%$ dos artigos focalizaram os determinantes do envolvimento direcionando-se às políticas sociais existentes voltadas para a paternidade, incluindo os programas de empregabilidade direcionados aos pais sem custódia (Bloomer \& Sipe, 2003). Ressaltam-se as implicações negativas das dificuldades econômicas vivenciadas pelos pais nas distintas faixas etárias, evidenciando a necessidade de estudos que permitam traçar um panorama destas questões sociais nas relações familiares.

\section{Consequências da paternidade}

Nesta categoria foram analisados 85 artigos, sendo 52 (15\%) trabalhos abordando o ponto de vista do pai e $33(9 \%)$ as consequências para o filho. As subcategorias são descritas a seguir:

(1) Consequências para o pai: os resumos nacionais $(n=12,13 \%)$ e internacionais $(n=40,15 \%)$ totalizaram 52 artigos que investigaram o significado e as consequências da paternidade, o impacto da gravidez e do nascimento do filho
(Johnson \& Baker, 2004), destacando o desenvolvimento da personalidade masculina na paternidade (Borisenko, 2007) e a maior proximidade com a família extensa (Knoester \& Eggebeen, 2006). Os artigos analisam o movimento "paternidade responsável" iniciado em 1990 (Gavanas, 2004) e como os modelos de paternidade se entrecruzam. O modelo tradicional de provedor coexiste com o novo modelo de pai cujo vínculo afetivo inicia desde a gestação (Puyana \& Mosquera, 2005), levando o homem a conciliar paternidade, casamento e trabalho (Coltrane, 2006) e a avaliar sua importância na vida das crianças frente às incertezas do século XXI (Bradley, Shears, Roggrnan, \& Tamis-LeMonda, 2006). A paternidade tardia é discutida numa reflexão sobre ser pai idoso (Colarusso, 2005) e como os homens de meia-idade percebem a si mesmos como pais (Carr, 2005). A paternidade como um componente da saúde é analisada em artigos sobre o sentido para viver construído após o nascimento dos filhos em pais portadores de HIV (Sherr \& Barry, 2004), como também o risco de desordem mental em pais no período pós-parto (Brennan, Ayers, Ahmed, \& Marshall-Lucette, 2007). As publicações nacionais procuraram compreender as demandas subjetivas advindas da responsabilidade assumida pelo homem com o nascimento do filho, conhecendo seus sentimentos e expectativas (Lopes, Menezes, Santos, \& Piccinini, 2006).

(2) Consequências para o filho: nesta categoria foram encontrados 19 artigos internacionais (8\%) e 34 artigos nacionais $(13 \%)$. Os trabalhos mostraram a influência positiva dos pais no desenvolvimento emocional de seus filhos (Lewis \& Lamb, 2003) e a percepção dos filhos adultos sobre a afeição transmitida por seus pais (Floyd, Sargent, \& Di Corcia, 2004). Além disto, alguns trabalhos destacaram as consequências dos maus-tratos dos pais sobre as crianças (Eriksson \& Hester, 2001; Waller \& Swisher, 2006). No Brasil, observaram-se publicações na área da Psicanálise (Teixeira, 2002) e temáticas envolvendo questões sociais como a repercussão do afastamento do pai no desenvolvimento dos filhos (Pereira \& Silva, 2006) e a repercussão dos valores laborais dos pais sobre os valores de realização e estabilidade profissional dos filhos (Porto \& Tamayo, 2006).

\section{Discussão}

Desde a década de 1970, quando Lamb discutiu a importância do papel paterno no desenvolvimento infantil, introduzindo a paternidade como um tópico relevante e pouco considerado nas investigações (Lamb, 1997), muitos trabalhos foram produzidos, resultando em contribuições importantes para a Psicologia e para os estudos sobre família. Desta maneira, considerando o objetivo principal deste artigo, a saber, realizar um levantamento bibliográfico das publicações sobre paternidade na última década, verificou-se que o tema permanece como um tópico presente na produção nacional e internacional associado a pesquisas em diversas áreas. Mesmo reconhecendo as limitações geradas pelo enquadramento 
de cada artigo em uma única categoria, os resultados refletem o centro das principais preocupações da comunidade acadêmica, permitindo identificar especificidades significativas na comparação entre a produção nacional e internacional.

No geral, observa-se que tanto a produção nacional como a internacional mantiveram-se constantes e crescentes ao longo dos últimos anos. Chama a atenção o grande número de trabalhos apoiados em delineamentos qualitativos, o que aponta para o interesse em conhecer a paternidade a partir das experiências individuais ou grupais percebidas pelo próprio sujeito. Apesar da maior parte dos trabalhos ter procedência norte-americana e européia, a paternidade foi tópico de pesquisa em diversos países e culturas. Neste sentido, a produção nacional foi significativa, e o Brasil destacou-se pelo número de trabalhos voltados para o tema. Considerando as características patriarcais da sociedade brasileira, o interesse pelo papel masculino na família aponta para uma posição de reflexão e questionamento das relações familiares, objetivando incentivar o envolvimento paterno na família.

A análise temática realizada pela classificação dos trabalhos nos eixos principais de investigação também permitiu a identificação dos principais focos de pesquisa. Tomando como referência os eixos caracterização, determinantes e consequências da paternidade, observa-se na esfera internacional um interesse por trabalhos voltados para os aspectos históricos, políticos e culturais. É evidente uma preocupação com questões relacionadas às esferas macrossociais que influenciam o exercício da paternidade, com destaque para os aspectos econômicos e sociais como impulsionadores do comportamento paterno. Mesmo que a noção da necessidade do envolvimento masculino com os filhos em diferentes âmbitos do desenvolvimento infantil e das relações familiares esteja altamente difundida, "o novo pai" não corresponde à realidade observada. Portanto, políticas e programas de apoio ao envolvimento paterno são considerados essenciais para a transformação e consolidação da responsabilidade masculina para com os filhos, refletindo-se em programas de saúde voltados para dar assistência às famílias de baixa renda, aos pais solteiros e presidiários, com o intuito de criar condições para o exercício saudável da paternidade.

Em nível nacional, o grande volume e constância de publicações demonstram a importância dada ao tema pela comunidade científica brasileira. A grande ênfase da discussão sobre as características da paternidade na adolescência, em contraposição à paternidade na idade adulta, por exemplo, reflete uma questão social importante do contexto brasileiro, em função do grande número de adolescentes grávidas e da implicação desta gestação tanto para a futura mãe como para o pai jovem. No Brasil, há menor ênfase nos programas voltados para a promoção da paternidade, denotando a precariedade de ações de assistência social mais consistentes no sentido de prevenir repercussões negativas decorrentes do afastamento/negligência da figura paterna no contato com as crianças. Percebe-se, neste sentido, que o afastamento do pai da práxis da paternidade é compreendido como decorrência da combinação de uma série de variáveis, incluindo desde a construção de sua personalidade, as condições relacionais e até as questões socioculturais.

As diversas configurações familiares contemporâneas são abordadas pelos cientistas sociais nacionais e internacionais, considerando os fatores que facilitam e dificultam a prática paterna. As pesquisas voltadas à inevitável limitação da paternidade na não-custódia indicam a importância do suporte familiar advindo da relação entre os ex-companheiros, assim como a importância da rede de relações que se estabelecem na construção do vínculo nas famílias reconstituídas ou monoparentais para a manutenção da responsabilidade paterna. Também, dentre os artigos publicados, alguns temas passam a adquirir contornos mais definidos, principalmente os da homoparentalidade, questionando as relações de gênero como fator excludente do processo de parentalidade. Assim, a análise das transformações no perfil e práxis familiares reflete um período de transição da figura paterna.

Um aspecto que também foi objeto significativo de estudo foi a avaliação do impacto das transformações do papel paterno na vivência do próprio pai, evidenciando a necessidade de entender estas dinâmicas como pressuposto para a construção de um novo sentido de paternidade. Portanto, além do impacto positivo do envolvimento masculino no desenvolvimento infantil, a paternidade foi descrita como um processo de grandes transformações para o homem, estando atrelada às condições sociais e pessoais do pai. Seguindo esta lógica de entendimento dos fatores que afetam significativamente a construção de um novo sentido de paternidade, $o$ levantamento deixa evidente a lacuna nas discussões nacionais sobre as variáveis socioeconômicas e a paternidade, especialmente diante do reconhecimento de que se vive numa sociedade onde as condições sociais têm impacto significativo nas questões familiares.

Entretanto, cabe destacar que a produção nacional, mesmo não consolidada em todos os aspectos associados às questões da paternidade, evidenciou diversidade de focos nas pesquisas, refletindo o interesse da comunidade acadêmica pelo impacto das questões contemporâneas na parentalidade. Tal é o caso de estudos nacionais voltados para grupos mais específicos, como adoção, maus-tratos, técnicas reprodutivas, pais portadores de HIV, entre outros. Assim, a pesquisa nacional reflete preocupação e reconhecimento da importância masculina na família, apontando áreas que prescindem de mais investigações. Pais contemporâneos transitam entre o modelo patriarcal identificado com a responsabilidade masculina de promover a estabilidade econômica de sua família e o desejo de maior envolvimento afetivo com sua mulher e filhos (Unbehaum, 2000). Justamente nestas questões reside a necessidade de desenvolvimento de estudos para a compreensão do universo masculino em relação à definição da masculinidade e do papel paterno face às exigências contemporâneas. 


\section{Considerações finais}

Em suma, o tema da paternidade ainda se constitui como foco de interesse importante para a compreensão das relações familiares e das condições de desenvolvimento infantil, questão fundamental para a implantação de políticas públicas de apoio e suporte às famílias em diferentes contextos. A pluralidade de temas associados à paternidade indica a complexidade de situações que determinam e facilitam o envolvimento masculino com os filhos. Porém, apesar desta diversidade de contextos e situações associadas à paternidade, todos os estudos avaliados neste trabalho foram unânimes na compreensão da importância do envolvimento e participação masculina no cuidado dos filhos. Neste sentido, a constatação central quanto às transformações da paternidade refere-se à importância de compreender as implicações das questões familiares contemporâneas com o intuito de identificar os entraves ao maior envolvimento masculino, de forma a propor ações, tanto em uma perspectiva individual quanto social, de incentivo, participação e reconhecimento da paternidade.

\section{Referências}

Almeida, A. F. F., \& Hardy, E. (2007). Gender vulnerability for parenthood among male adolescents. Revista de Saúde Pública, 41, 565-572.

Anderson, E. A., Kohler, J. K., \& Letiecq, B. L. (2002). Lowincome fathers and responsible fatherhood programs: A qualitative investigation of participants' experiences. Family Relations, 51, 148-155.

Anderson, E. A., Kohler, J. K., \& Letiecq, B. L. (2005). Predictors of depression among low income, nonresidential fathers. Journal of Family Issues, 26, 547-567.

Anderson, D. A., \& Hamilton, M. (2005). Gender role stereotyping of parents in children's picture books: The invisible father. Sex Roles, 52, 145-151.

Andrade, R. P., Costa, N. R. A., \& Rossetti-Ferreira, M. C. (2006). Significações de paternidade adotiva: Um estudo de caso. Paidéia (Ribeirão Preto), 16, 241-252.

Arenas, M. L., \& Greif, G. L. (2000). Issues of fatherhood and recovery for VA substance abuse patients. Journal Psychoactive Drugs, 32, 339-41.

Aronson, R. E., Whitehead, T. L., \& Baber, W. L. (2003). Challenges to masculine transformation among urban low-income african american males. American Journal of Public Health, 93, 732-741.

Balancho, L. S. F. (2004). Ser pai: Transformações intergeracionais na paternidade. Análise Psicológica, 2, 377-386.

Baum, N. (2004). Coping with absence-presence: Noncustodial fathers' parenting behaviors. American Journal of Orthopsychiatry, 74, 316-324.

Bem, L. A., \& Wagner, A. (2006). Reflexões sobre a construção da parentalidade e o uso de estratégias educativas em famílias de baixo nível socioeconômico. Psicologia em Estudo, 11, 63-71.
Bigras, M., \& Paquette, D. (2000). A interdependência entre os subsistemas parental e marital: Uma análise da pessoa-processo-contexto. Psicologia: Teoria e Pesquisa, 16, 91-102.

Bishop, V., Gay, F., Ledesma, J., \& Garofalo, R. (2005). Exploring the adolescent and young adult minority fatherhood experience. Journal of Adolescent Health, 36, 119-120.

Bloomer, S. R, \& Sipe, T. A. (2003). The impact of the Georgia fatherhood program on employment and wages. Journal of Social Service Research, 29(4), 53-65.

Borisenko, J. (2007). Fatherhood as a personality development factor in men. Spanish Journal of Psychology, 10, 82-90.

Bradley, R. H., Shears, J. K., Roggrnan, L. A., \& Tamis-LeMonda, C. S. (2006). Lessons learned from early head start for fatherhood research and program development. Parenting: Science and Practice, 6, 259-271.

Brasileiro, R. F., Jablonski, B., \& Féres-Carneiro, T. (2002). Papéis de gênero, transição para paternidade e a questão da tradicionalização. Psico, 33, 289-310.

Brennan, A., Ayers, S., Ahmed, H., \& Marshall-Lucette, S. (2007). A critical review of the couvade syndrome: The pregnant male. Journal of Reproductive and Infant Psychology, 3, 173-189.

Brito, L. M. T. (2005). De papai sabe tudo a como educar seus pais: Considerações sobre programas infantis de TV. Psicologia e Sociedade, 17, 17-28.

Bustamante, V. (2005). Ser pai no subúrbio ferroviário de Salvador: Um estudo de caso com homens de camadas populares. Psicologia em Estudo, 10, 393-402.

Bygren, M., \& Duvander, A. Z. (2006). Parents' workplace situation and fathers' parental leave use. Journal of Marriage and the Family, 68, 363-372.

Cabral, C. S. (2003). Contracepção e gravidez na adolescência na perspectiva de jovens pais de uma comunidade favelada do Rio de Janeiro. Caderno de Saúde Pública, 19, 283-92.

Cabrera, N. J., Tamis-LeMonda, C. S., Bradley, R. H., Hofferth, S., \& Lamb, M. E. (2000). Fatherhood in the twenty-first century. Child Development, 71, 127-136.

Cabrera, N., \& Peters, H. E. (2000). Public policies and father involvement. Marriage and Family Review, 29, 295314.

Carr, D. (2005). The psychological consequences of midlife men's social comparisons with their young adult sons. Journal of Marriage and the Family, 67, 240-250.

Carvalho, M. L. M. (2003). Participação dos pais no nascimento em maternidade pública: Dificuldades institucionais e motivações dos casais. Cadernos de Saúde Pública, 19, 389-398.

Colarusso, C. A. (2005). The evolution of paternal identity in late adulthood. Journal of the American Psychoanalytic Association, 53, 51-81. 
Coltrane, S. (2006). Book review: The package deal: Marriage, work and fatherhood in men's lives. Men and Masculinities, 8, 380-381.

Dantas, C., Jablonski, B., \& Féres-Carneiro, T. (2004). Paternidade: Considerações sobre a relação pais-filhos após separação conjugal. Paidéia (Ribeirão Preto), 14, 347-357.

Davies, B., Gudmundsdottir, M., Worden, B., Orloff, S., Sumner, L., \& Brenner, P. (2004). Living in the dragon's shadow fathers' experiences of a child's life-limiting illness. Death Studies, 28, 111-135.

Dermott, E. (2003). The intimate father: Defining paternal involvement [Versão eletrônica]. Sociological Research Online, 8(4). Recuperado em 15 dezembro 2007, de http:// www.socresonline.org.uk/8/4/dermott.html

Dias, A. B., \& Aquino, E. M. L. (2006). Maternidade e paternidade na adolescência: Algumas constatações em três cidades do Brasil. Cadernos de Saúde Pública, 22, 14471458.

Eggebeen, D. J., \& Knoester, C. (2001). Does fatherhood matter for men? Journal of Marriage and the Family, 63, 381-393.

Eriksson, M., \& Hester, M. (2001). Violent men as goodenough fathers? A look at England and Sweden. Violence Against Women, 7, 779-798.

Farinati, D. M., Rigoni, M. S., \& Müller, M. C. (2006). Infertilidade: Um novo campo da psicologia da saúde. Estudos de Psicologia (Campinas), 23, 433-439.

Floyd, K., Sargent, J. E., \& Di Corcia, M. (2004). Human affection exchange: VI. Further tests of reproductive probability as a predictor of men's affection with their adult sons. Journal of Social Psychology, 144, 191-206.

Futino, R. S., \& Martins, S. (2006). Adoção por homossexuais: Uma nova configuração familiar sob os olhares da psicologia e do direito. Aletheia, 24, 149-159.

Gaertner, B. M., Spinrad, T. L., Eisenberg, N., \& Greving, K. A.. (2007). Parental childrearing attitudes as correlates of father involvement during infancy. Journal of Marriage and the Family, 69, 962-976.

Gavanas, A. (2004). Domesticating masculinity and masculinizing domesticity in contemporary US fatherhood politics. Social Politics, 11, 247-266.

Gomes, A. J. S., \& Resende, V. R. (2004). O pai presente: O desvelar da paternidade em uma família contemporânea. Psicologia: Teoria e Pesquisa, 20, 119 -125.

Gonzalez-Lopez, G. (2004). Fathering latina sexualities: Mexican men and the virginity of their daughters. Journal of Marriage and the Family, 66, 1118-1130.

Gray, A. (2006). The time economy of parenting [Versão eletrônica]. Sociological Research Online, 11. Recuperado em 11 março 2008, de http://www.socresonline.org. uk/11/3/gray.html

Gupta, S., Smock, P. J., \& Manning, W. D. (2004). Moving out: Transition to nonresidence among resident fathers in the United States, 1968-1997. Journal of Marriage and the Family, 66, 627-638.
Hairston, C. F. (2002). Fathers in prison: Responsible fatherhood and responsible public policies. Marriage and Family Review, 32(3-4), 111-135.

Halford, S. (2006). Collapsing the boundaries? Fatherhood, organization and home-working. Gender Work and Organization, 13, 383-402.

Heilborn, M. L., Salem, T., Rohden, F., Brandão, E., Knauth, D., Víctora, C., Aquino, E., McCallum, C., \& Bozon, M. (2002). Aproximações socioantropológicas sobre a gravidez na adolescência. Horizontes Antropológicos, 8 , 13-45.

Hennigen, I., \& Guareschi, N. M. F. (2002). A paternidade na contemporaneidade: Um estudo de mídia sob a perspectiva dos estudos culturais. Psicologia e Sociedade, 14, 44-68.

Joseph, L. (2006). Fatherhood politics in the United States. Men and Masculinities, 8, 528-529.

Johnson, M. P., \& Baker, S. R. (2004). Implications of coping repertoire as predictors of men's stress, anxiety and depression following pregnancy, childbirth and miscarriage: A longitudinal study. Journal of Psychosomatic Obstetrics and Gynecology, 25, 87-98.

Kao, C. H., \& Long, A. (2004). First-time taiwanese expectant fathers' life experiences during the third trimester of pregnancy. Journal of Nursing Research, 12, 60-71.

Kiernan, K. (2006). Non-residential fatherhood and child involvement: Evidence from the millennium cohort study. Journal of Social Policy, 35, 651-669.

Knoester, C., \& Eggebeen, D. J. (2006). The effects of the transition to parenthood and subsequent children on men's well-being and social participation. Journal of Family Issues, 27, 1532-1560.

Lamb, M. E. (Ed.). (1997). The role of the father in child development. New York: John Wiley \& Sons.

Lamb, M. E. (2000). Exploring and defining early social ecologies and their impact: Mothers, fathers, families, and cultures. Marriage and Family Review, 30, 119-135.

LaRossa, R. (2004). The culture of fatherhood in the fifties: A closer look. Journal of Family History, 29, 47-70.

Lewis, C., \& Lamb, M. E. (2003). Fathers' influences on children's development: The evidence from two-parent families. European Journal of Psychology of Education, 18, 211-228.

Lopes, R. C. S., Menezes, C., Santos, G. P., \& Piccinini, C. A. (2006). Ritual de casamento e planejamento do primeiro filho. Psicologia em Estudo, 11, 55-61.

Lubbe, C. (2007). Mothers, fathers, or parents: Samegendered families in South Africa. South African Journal of Psychology, 37, 260-283.

MacMillan, C. (2005). Public health initiative at a young offenders institute. Community Practitioner, 78, 397-399.

McVeigh, C. A., Baafi, M., \& Williamson, M. (2002). Functional status after fatherhood: An australian study. Journal of Obstetric Gynecologic and Neonatal Nursing, 31, 165-171. 
Madsen, S. A., Lind, D., \& Munck, H. (2007). Men's abilities to reflect their infants' states of mind: Interviews with 41 new fathers on experiences of parenthood. Nordic Psychology, 59, 149-163.

Manning, W. D., \& Smock, P. J. (2000). Swapping families: Serial parenting and economic support for children. Journal of Marriage and the Family, 62, 111-122.

Marsiglio, W., \& Hutchinson, S., \& Cohan, M. (2000). Envisioning fatherhood: A social psychological perspective on young men without kids. Family Relations, 49, 133-142.

Marsiglio, W., Day, R. D., \& Lamb, M. E. (2000). Exploring fatherhood diversity: Implications for conceptualizing father involvement. Marriage and Family Review, 29, 269-293.

Marsiglio, W., \& Hinojosa, R. (2007). Managing the multifather family: Stepfathers as father allies. Journal of Marriage and the Family, 69, 845-862.

Masciadrelli, B. P., Pleck, J. H., \& Stueve J. L. (2006). Fathers' role model perceptions: Themes and linkages with involvement. Men and Masculinities, 9, 23-34.

Motta, C. C. L., \& Crepaldi, M. A. (2005). O pai no parto e apoio emocional: A perspectiva da parturiente. Paidéia (Ribeirão Preto), 15, 105-118.

Narvaz, M. G., \& Koller, S. H. (2006). Famílias e patriarcado: Da prescrição normativa à subversão criativa. Psicologia \& Sociedade, 18, 49-55.

Nelson, T. J. (2004). Low-income fathers. Annual Review of Sociology, 30, 427-451.

Neves, J. L. (1996). Pesquisa qualitativa: Características, usos e possibilidades. Cadernos de Pesquisas em Administração, $1,1-5$.

Paiva, V., Lima, T. M., Santos, N., Ventura-Filipe, E., \& Segurado, A. (2002). Sem direito de amar? A vontade de ter filhos entre homens (e mulheres) vivendo com o HIV. Psicologia USP, 13, 105-133.

Passos, M. C. (2005). Homoparentalidade: Uma entre outras formas de ser família. Psicologia Clínica, 17, 31-40.

Perala-Littunen, S. (2007). Gender equality or primacy of the mother? Ambivalent descriptions of good parents. Journal of Marriage and the Family, 69, 341-351.

Pereira, R. C., \& Silva, C. M. (2006). Nem só de pão vive o homem. Sociedade e Estado, 21, 667-680.

Peterson, G. W., \& Steinmetz, S. K. (2000). The diversity of fatherhood: Change, constancy, and contradiction. Marriage and Family Review, 29, 315-322

Pleck, J. H. (1997). Paternal involvement: Levels, sources, and consequences. In M. E. Lamb (Eds.), The role of the father in child development. New York: John Wiley \& Sons.

Pohlman, S. (2005). The primacy of work and fathering preterm infants: Findings from an interpretive phenomenological study. Advances in Neonatal Care, 5, 204-216.

Porto, J. B., \& Tamayo, A. (2006). Influência dos valores laborais dos pais sobre os valores laborais dos filhos. Psicologia: Reflexão e Crítica, 19, 151-158.
Puyana, Y., \& Mosquera, C. (2005). Traer hijos o hijas al mundo: Significados culturales de la paternidad y la maternidad. Revista Latinoamericana de Ciencias Sociais, Niñez y Juventud, 3, 111-140.

Rocha, L. E., \& Debert-Ribeiro, M. (2001). Work and health: A gender study on systems analysts. Revista de Saúde, 35, 539-547.

Santos, M. C., Caldana, R. H. L., \& Biasoli-Alves, Z. M. M. (2001). O papel masculino dos anos quarenta aos noventa: Transformações no ideário. Paidéia (Ribeirão Preto), $11,57-68$.

Schindler, H. S., \& Coley, R. L. (2007). A qualitative study of homeless fathers: Exploring parenting and gender role transitions. Family Relations, 56, 40-51.

Serafim, D., \& Lindsey, P. C. (2002). O aleitamento materno na perspectiva do pai. Ciência, Cuidado e Saúde, 1, 23-27.

Seward, R. R. (2003). The emperor's embrace: The evolution of fatherhood. Family Relations, 52, 309-310.

Sherr, L., \& Barry, N. (2004). Fatherhood and HIV-positive heterosexual men. HIV Medicine, 5, 258-263.

Silva, M. R., \& Piccinini, C. A. (2004). O envolvimento paterno em pais não-residentes: Algumas questões teóricas. Psico, 35, 185-194.

Skevik, A. (2006). Absent fathers or reorganized families? Variations in father-child contact after parental break-up in Norway. Sociological Review, 54, 114-132.

Teixeira, L. C. (2002). Função paterna, fratria e violência: Sobre a constituição do socius na psicanálise freudiana. Psico USF, 7,195-200.

Thurler, A. L. (2004). Paternidade e deserção: Crianças sem reconhecimento, maternidades penalizadas pelo sexismo. Sociedade e Estado, 19, 501-502.

Thurler, A. L. (2006). Outros horizontes para a paternidade brasileira no século XXI? Sociedade e Estado, 3, 681-707.

Trindade, Z. A., \& Menandro, M. C. S. (2002). Pais adolescentes: Vivência e significação. Estudos de Psicologia (Natal), 7, 15-23.

Thomas, M., \& Bailey, N. (2006). Square pegs in round holes? Leave periods and role displacement in UK-based seafaring families. Work Employment and Society, 20, 129-149.

Tronchin, D. M. R., \& Tsunechiro, M. A. (2006). Cuidar e o conviver com o filho prematuro: A experiência do pai. Revista Latinoamericana de Enfermagem, 14, 93-101.

Tudge, J., Hayes, S., Doucet, F., Odero, D., Kulakova, N., Tammeveski, P., Meltsas, M., \& Lee, S. (2000). Parent's participation in cultural practices with their preschoolers. Psicologia: Teoria e Pesquisa, 16, 1-10.

Tudge, J., Piccinini, C. A., Gonçalves, T. R., Lopes R. S., \& Silva, M. R. (2004). O Envolvimento paterno durante a gestação. Psicologia: Reflexão e Crítica, 17, 303-314.

Unbehaum, S. G. (2000). Experiência masculina da paternidade nos anos 1990: Estudo de relações de gênero com homens de camadas médias. Dissertação de mestrado não-publicada, Faculdade de Filosofia, Letras e Ciências Humanas, Universidade de São Paulo, São Paulo. 
Wall, G., \& Arnold, S. (2007). How involved is involved fathering? An exploration of the contemporary culture of fatherhood. Gender \& Society, 21, 508-527.

Walker, A. J., \& McGraw, L. A. (2000). Who is responsible for responsible fathering? Journal of Marriage and the Family, 62, 563-569.

Waller, M. R., \& Swisher, R. (2006). Fathers' risk factors in fragile families: Implications for healthy relationships and father involvement. Social Problems, 53, 392-420.

Wei, E. H., Loeber, R., \& Stouthamer-Loeber, M. (2002). How many of the offspring born to teenage fathers are produced by repeated serious delinquents? Criminal Behavior and Mental Health, 12, 83-98.

Whitehouse, G. (2002). Parenthood and pay in Australia and the UK: Evidence from workplace surveys. Journal of Sociology, 38, 381-397.

Carmen Lúcia Carvalho de Souza é Professora Titular do curso de Psicologia da Universidade Comunitária Regional de Chapecó.

Silvia Pereira da Cruz Benetti é Professora Adjunta do curso de Psicologia e do Programa de Pós-graduação em Psicologia da Universidade do Vale do Rio dos Sinos.

Recebido: $15 / 05 / 2008$

$1^{a}$ revisão: 16/01/2009

Aceite final: 05/02/2009 